\title{
Rendimiento académico e inserción laboral de los titulados en medicina por la Universitat de Barcelona. Un estudio longitudinal
}

\author{
Begoña Campos, Berenguer Camps, Arelly Ornelas, Roser Cussó, Albert Torras, Pilar Arrizabalaga
}

Introducción. El proceso de Bolonia ha provocado cambios en la estructura universitaria dando protagonismo a los conceptos de competencia y calidad. Esta situación planteó el reto de querer mostrar la contribución de la Universitat de Barcelona (UB) en la formación de médicos para la sociedad catalana y la distribución de estos titulados en relación a todo el colectivo de profesionales colegiados en la provincia de Barcelona.

Sujetos y métodos. Estudio longitudinal de cuatro promociones de alumnos de la Facultad de Medicina de la UB, que ingresaron entre 1994 y 2001. Para cada cohorte se calcularon estadísticas de rendimiento académico, de formación especializada y de colegiación después de consultar diferentes bases de datos y utilizando el programa informático R.

Resultados. El 85-96\% de los alumnos que ingresaron en la Facultad de Medicina obtuvieron el título de licenciado en un plazo inferior a siete años. Del total de licenciados, un $83 \%$ constaba registrado en el colegio oficial de médicos de la provincia (COMB). Comparadas con la población colegiada de profesionales, estas promociones destacan por una tasa de feminización mayor (tres de cada cuatro) y tasas de extranjería prácticamente nulas.

Conclusiones. Los titulados en medicina por la UB demostraron un alto rendimiento de estudio y se insertaron a la profesión en su entorno geográfico.

Palabras clave. Aseguramiento de la calidad. Competencia profesional. Educación médica. Eficiencia académica.

Academic achievement and work insertion of Barcelona University medical graduates. A longitudinal study

Introduction. The Bologna process has brought about changes in the structure of the Spanish University giving prominence to the concepts of competence and quality. This posed the challenge of showing the contribution of the University of Barcelona (UB) in the training of doctors to Catalan society, and the distribution of these graduates in relation to the entire group of professional associations in the province of Barcelona.

Subjects and methods. Longitudinal study of four classes of freshman students of the Faculty of Medicine, UB, who were admitted between 1994 and 2001. For each cohort academic performance, specialized training and licensing statistics were obtained after looking up different databases and using the R software.

Results. Between $85 \%$ and $96 \%$ of students who entered medical school obtained a degree in Medicine in a period shorter than seven years. Of all graduates comprised $83 \%$ recorded in medical professional association of the province (COMB). In comparison with the total professional members, these classes highlighted by a greater proportion of women ( 3 out of 4 ) and virtually no immigration rates.

Conclusions. Graduates in Medicine from the UB demonstrated high performance of their studies and inserted into the profession in its geographical area.

Key words. Academic organizational efficiency. Medical education. Professional competence. Quality assurance.

\section{Introducción}

En la última década, la universidad española ha sufrido un cambio de su marco legal y organizativo que se conoce como proceso de Bolonia [1]. Es la consecuencia de una apuesta europea por impulsar una sociedad basada en el conocimiento, reflejada en 1999 en la Declaración de Bolonia, que define un Espacio Europeo de Educación Superior (EEES) para implicar así a las universidades como agentes activos en el proceso de convergencia [2].

El proceso de Bolonia ha provocado, durante el diseño de los nuevos planes de grado, una intensa reflexión sobre la finalidad de la universidad que ha
Departamento de Salud Pública (B. Campos, A. Ornelas); Departamento de Ciencias Fisiológicas (R. Cussó): Departamento de Medicina (A. Torras); Facultad de Medicina; Universitat de Barcelona. Gabinet d'Estudis Colegials; Collegi Oficial de Metges de Barcelona (B. Camps). Consultora de Nefrología; Hospital Clínic; IDIBAPS (P. Arrizabalaga). Secretaria General; Junta de Gobierno; Collegi Oficial de Metges de Barcelona (P. Arrizabalaga). Barcelona, España.

Correspondencia:

Dra. Pilar Arrizabalaga. Secretaria General. Junta de Gobierno. Colllegi Oficial de Metges de Barcelona (COMB). Pg. Bonanova, 47. E-08017 Barcelona.

Fax: +34935678899.

E-mail:

secretaria_junta@comb.cat

Agradecimientos:

Fue fundamental la colaboración de la Secretaría de Estudiantes y Docencia de la Facultad de Medicina de la Universitat de Barcelona (UB), así como de la Unidad de Recursos de Informática del COMB. F. Delgado puso en marcha los primeros programas para el análisis de datos y junto a M.C. Eraso, que buscó y ordenó los datos de MIR, generaron el primer informe provisional de resultados. A. Ornelas continuó el trabajo hasta la obtención de los resultados finales. Los tres disfrutaron de becas de colaboración de la UB durante el tiempo que participaron en el proyecto.

Conflicto de intereses: No declarado.

Competing interests: None declared.

(c) 2015 FEM 
dado relevancia al concepto de competencia [3]. Si la reforma está orientada a nuevos modelos de producción para mejorar la inserción laboral, la formación universitaria debería considerar el perfil profesional que demandan los cambios sociales $[4,5]$.

En el caso de medicina, este debate ha puesto de manifiesto la necesidad de mejorar algunos aspectos como la competencia comunicativa del médico con el paciente o el compromiso con el sistema sanitario en la toma de decisiones [6,7]. En la prácti$\mathrm{ca}$, este proceso de reforma afecta poco a los contenidos y no altera la duración de la formación de pregrado, que se mantiene en seis cursos. Los esfuerzos se han centrado en adaptar un sistema de créditos académicos para facilitar la movilidad estudiantil [8] y especialmente en adaptar las metodologías para que el estudio esté centrado en el trabajo del estudiante [9].

Este nuevo marco contribuye a introducir en el sistema educativo la cultura de la calidad para evaluar sus instituciones. Las universidades son las responsables a partir de ahora de demostrar la eficacia de sus procesos de garantía interna de la calidad utilizando criterios comunes a toda Europa [10]. Esta situación lleva a plantearse la eficiencia de cada centro en la formación de sus estudiantes [11] y cuál es su inserción profesional posterior.

El objetivo principal del proyecto fue aportar datos sobre la contribución de la Universitat de Barcelona (UB) en la formación de médicos para la sociedad catalana. También se quería estudiar la tendencia profesional de los licenciados en la Facultad de Medicina de la UB en el contexto de la población colegiada en el Col-legi Oficial de Metges de Barcelona (COMB). Los resultados obtenidos deberían servir como referente para evaluar los beneficios de la reforma de Bolonia. Un objetivo secundario fue analizar las características demográficas (sexo y procedencia) del alumnado en relación con el conjunto de médicos colegiados en el COMB.

\section{Sujetos y métodos}

Se diseñó un estudio descriptivo de cuatro promociones de estudiantes de la Facultad de Medicina de la UB, entendiendo como promoción la cohorte de individuos que iniciaron sus estudios el mismo curso académico. Para cada promoción se realizó un análisis longitudinal retrospectivo, con seguimiento individual, abarcando desde el inicio de la licenciatura hasta la finalización del posgrado.

La selección de las promociones se realizó teniendo en cuenta dos criterios: que hubieran acabado antes de realizar el estudio y que hubieran iniciado estudios después de 1994, ya que en ese año se puso en marcha un nuevo plan de estudios que se reformó luego en 2001. Esto delimitó el marco temporal y las cuatro promociones se eligieron de forma que quedaran repartidas homogéneamente dentro de él.

A partir de actas en papel de asignaturas de primer semestre se identificó a los integrantes de cada promoción. Esto permitió buscar en extractos de la base de datos de gestión académica para conseguir datos desagregados a partir de los cuales se elaboraron los resultados. Además, se realizaron consultas en la base de datos del COMB para conocer los destinos profesionales.

Se consultó el portal de estadísticas académicas de la UB para cotejar y complementar nuestros datos [12] y la página web del Ministerio de Sanidad para obtener resultados de las pruebas de acceso a formación sanitaria especializada (MIR) [13].

Para cada estudiante se calculó la nota de expediente utilizando las calificaciones de las 44 asignaturas obligatorias del plan de estudios de 2001, que suman 431,5 créditos del total de 500 . Este criterio permite hacer comparaciones evitando la variabilidad que generan los créditos optativos y de libre elección, y es similar al que se aplica en las pruebas MIR. Para las cohortes de 1994 y 1999 se aplicó la tabla de conversiones estandarizada. La nota final de expediente se obtuvo aplicando la equivalencia del sistema universitario español: 1 , aprobado; 2 , notable; 3 , sobresaliente, y 4 , matrícula de honor; y ponderado por los créditos de cada asignatura:

$\left[1 * c r e d \_A+2 * c r e d \_N+3 *\right.$ cred_E $+4{ }^{*}$ cred_MH $]$ Nota expediente $=\frac{\text { Total créditos }}{\text { To }}$

Para validar el procedimiento de cálculo se contrastaron algunos resultados con la nota oficial del expediente, comprobándose la concordancia de ambas fuentes. La gestión de los datos y la explotación estadística se realizó utilizando el programa $R$ [14]. Todos los datos personales se manejaron con arreglo a la Ley Orgánica de Protección de Datos.

\section{Resultados}

Los resultados obtenidos se presentan en dos bloques. En el primero se describe la población de universitarios que forman las promociones seleccionadas y su rendimiento, y en el segundo se comparan los médicos formados en la UB, pertenecientes a 
estas promociones, con el resto de la población colegiada en el COMB según sexo y procedencia.

\section{Características de los alumnos \\ titulados en medicina en la UB}

\section{Alumnos de nuevo ingreso}

El número de alumnos nuevos efectivamente matriculados en primer curso en cada promoción (Tabla I) fue ligeramente inferior a 240, numerus clausus fijado por la Generalitat de Catalunya.

Los estudios de medicina en la UB encabezan desde hace años la lista de las notas de corte más altas. La nota mínima de acceso a estos estudios por la vía de las pruebas de acceso a la universidad (PAU, alumnos de bachillerato) creció desde un 6,10 en 1990 hasta 8,74 en 2009. A esta mayoría de alumnos se fueron incorporando alumnos de ciclos formativos de grado superior, por la vía de acceso correspondiente, y cada vez con mejores expedientes académicos (9,06 en 2009).

\section{Tasa de licenciados}

En las cuatro promociones estudiadas, la tasa de alumnos que obtuvieron el título ha variado entre el 85\% y el 96\% (84-95\% según estadísticas oficiales de la UB) (Tabla I). Por consiguiente, las tasas de abandono fueron muy bajas, entre el 3,9\% y el 13,2\% según nuestros datos (3,5-13,8\% según estadísticas oficiales de la UB). La mitad de los abandonos ocurrieron durante el primer año, sin relación con la nota de acceso ni grandes diferencias entre hombres y mujeres. La tercera promoción fue la que tuvo mayor porcentaje de abandonos, especialmente varones.

El porcentaje de licenciados que completaron su formación en los seis cursos programados varía entre el $78 \%$ (1994 y 2001) y el 88\% (1999). El tiempo medio en todo caso fue inferior a siete años $(6,2-6,4$ años).

La distribución de las notas de expediente por promociones resultó bastante homogénea, sobre todo en la categoría de notable. que está en torno al 40\%. Cabe destacar la promoción que inició en 1996, con un $23 \%$ de sobresalientes. Las notas de segundo ciclo fueron siempre, por promoción y sexo, superiores a las de primer ciclo.

\section{Tasa de colegiación}

La mayoría de los licenciados de estas promociones desarrollaban su actividad profesional en la provincia de Barcelona en el momento del estudio. Sobre el total de 822 licenciados, un $83 \%$ constaba colegiado en el COMB en el momento del estudio y otro
Figura 1. Evolución de las nuevas colegiaciones según universidad de formación, entre 2000 y 2009. Porcentaje de titulados de la UB, del resto de universidades catalanas, del resto de España y extranjeras.

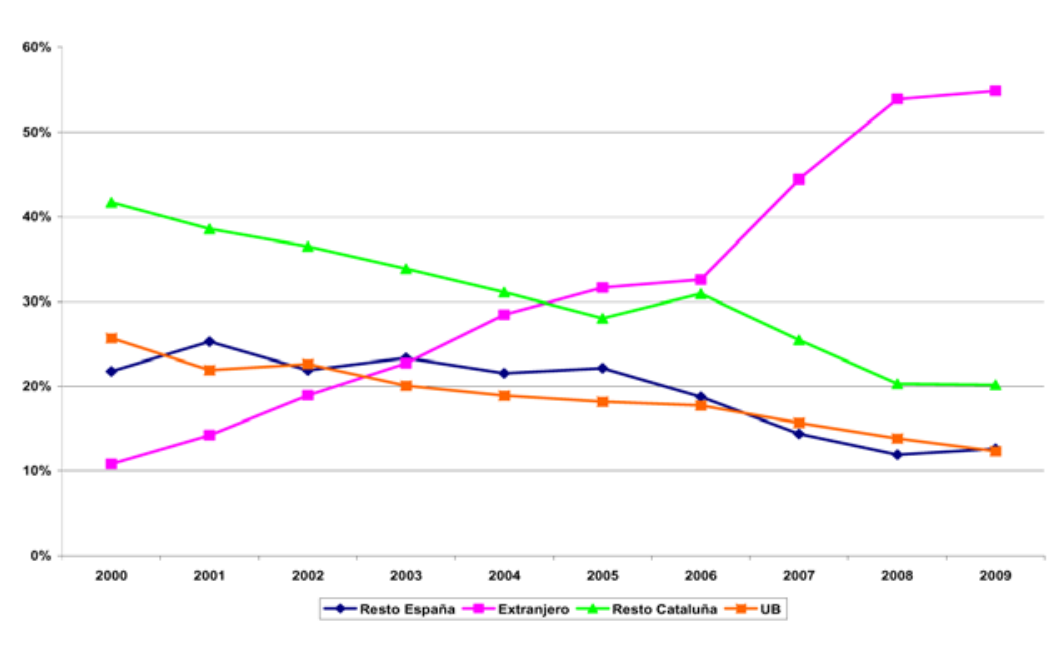

Tabla I. Estadísticas de las promociones estudiadas.

\begin{tabular}{|c|c|c|c|c|c|}
\hline \multirow{2}{*}{$\begin{array}{l}\text { Año de } \\
\text { inicio de la } \\
\text { promoción }\end{array}$} & \multicolumn{5}{|c|}{ Número de alumnos } \\
\hline & Nuevo ingreso ${ }^{a}$ & Identificados ${ }^{b}$ & Abandonos & Titulados & Colegiados COMB \\
\hline 1994 & 227 & 227 & 21 & $206(91 \%)$ & 193 (94\%) \\
\hline 1996 & 228 & 232 & 9 & $223(96 \%)$ & $181(81 \%)$ \\
\hline 1999 & 224 & 220 & 29 & $187(85 \%)^{c}$ & 159 (85\%) \\
\hline 2001 & 238 & 234 & 16 & $206(87 \%) c$ & $100(70 \%) d$ \\
\hline
\end{tabular}

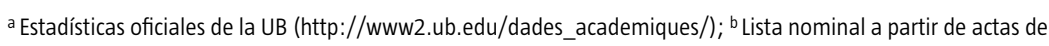
la cohorte de seguimiento; ${ }^{C}$ Casos confirmados en el momento del estudio, quedan aún alumnos pendientes de acabar; ${ }^{\mathrm{d}}$ Datos limitados al campus de Casanova $(n=143)$.

$6 \%$ lo había estado en el pasado. Se observa que la tasa de colegiación ha ido decreciendo: del $94 \%$ en la promoción de 1994 al $70 \%$ en la promoción de 2001 (Tabla I).

Asimismo, la contribución porcentual de los titulados por la UB a las nuevas colegiaciones año a año en la provincia de Barcelona ha disminuido en la última década (Fig. 1). En 2009 se situó en el 12\%, a la vez que crecían los colegiados de origen y formación extracomunitarios. Por lo tanto, aun no variando la aportación absoluta de la UB, su aportación porcentual desciende, igual que sucede con los titulados de otras universidades del resto de Cataluña y de España. 
Figura 2. Distribución por especialidades médicas y quirúrgicas según universidad de licenciatura: UB y resto de universidades catalanas.

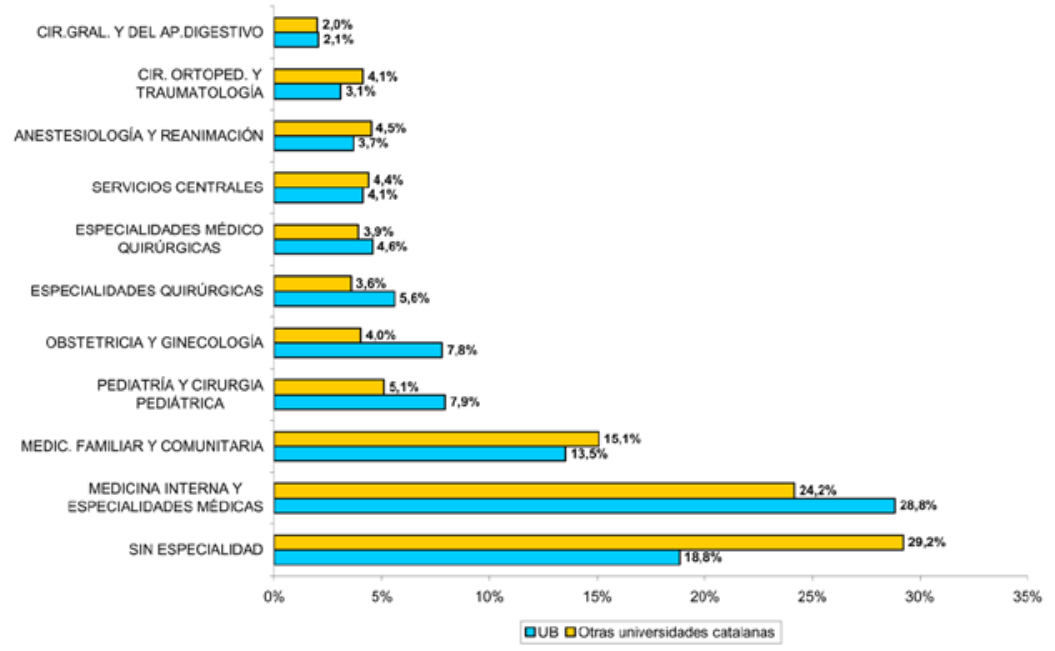

Tabla II. Especialidades que captan mayor proporción de alumnos licenciados.

\begin{tabular}{|c|c|c|c|c|}
\hline & 1994 & 1996 & 1999 & 2001 \\
\hline Medicina familiar y comunitaria & 32 & 31 & 11 & 8 \\
\hline Pediatría & 13 & 13 & 15 & 11 \\
\hline Obstetricia y ginecología & 13 & 14 & 14 & 13 \\
\hline Psiquiatría & 6 & 7 & 7 & 14 \\
\hline Medicina interna & $3^{a}$ & 11 & 15 & $3^{a}$ \\
\hline Cirugía ortopédica y traumatología & 5 & $1^{\mathrm{a}}$ & $4^{a}$ & $5^{a}$ \\
\hline $\begin{array}{l}\text { Porcentaje que agrupan las } \\
\text { especialidades más frecuentes }\end{array}$ & $52 \%(138)$ & $57 \%(135)$ & $49 \%(134)$ & $52 \%(104)^{b}$ \\
\hline Porcentaje de licenciados que inician MIR & $67 \%(206)$ & $61 \%(223)$ & $72 \%(187)$ & $50 \%(206)^{b}$ \\
\hline
\end{tabular}

a Especialidad poco frecuente, pero incluida a efectos de comparación; ' ${ }^{b}$ Fuente: página web del Ministerio de Sanidad.

\section{Perfil de especialidades}

El análisis de la formación médica especializada se vio limitado por la dificultad de acceder a los datos primarios. Los datos reunidos señalan que como mínimo un $60 \%$ de los licenciados acceden a la formación especializada (Tabla II).
Se observa que hay cuatro especialidades médicas que para todas las promociones son más prevalentes: medicina familiar y comunitaria, pediatría, obstetricia y ginecología, y psiquiatría (Tabla II). Al extender la lista para englobar al 50\%, entonces se incluiría medicina interna en las promociones de 1996 y 1999.

Cirugía cardiovascular, neurocirugía y cardiología son las especialidades elegidas por los alumnos con mejores expedientes de cada promoción, aparte de las anteriores. En cambio, la especialidad más prevalente, medicina familiar, no aparece entre las preferidas por estos alumnos.

Entre los colegiados en el COMB se observan diferencias entre la elección de especialidad por parte de los titulados en la UB y los titulados por otras universidades catalanas (Fig. 2). El número de médicos sin especialidad registrada es menor entre los titulados de la UB $(18,8 \%)$ que en el resto $(29,2 \%)$. Otra diferencia es la mayor presencia de titulados de la UB en medicina interna y especialidades médicas $(28,8 \%$ frente a $24,2 \%)$ y en obstetricia y ginecología (7,8\% frente a $4 \%)$.

\section{Composición de los titulados por sexo} y procedencia y comparación con el resto de la población colegiada en el COMB

\section{Tasa de feminización}

La presencia de mujeres al inicio y al final de los estudios es muy similar entre promociones: la tasa de abandono es muy baja y se reparte de forma bastante equilibrada entre sexos. En la figura 3 se presenta la distribución por sexos de cada promoción, comparándolos con la tasa de feminización del conjunto de los colegiados del COMB en esos mismos años. Alrededor del 75\% de los titulados en las promociones estudiadas son mujeres, y este porcentaje se reduce al $40 \%$ entre el conjunto de profesionales.

\section{Tasa de extranjería}

La prevalencia de extranjeros entre los alumnos de nuevo acceso fue inferior al $2 \%$ hasta la promoción de 2001, cuando se incrementa al 4\% (Fig. 4a).

En cuanto a los datos para el conjunto del COMB, se evidencia una incorporación más importante de médicos de nacionalidad extranjera, que pasa del 2,7\% en 1994 al 7,2\% en 2001 (Fig. 4b).

\section{Discusión}

Los resultados muestran un alto rendimiento de las cuatro promociones de medicina estudiadas. Los 
Figura 3. Tasa de feminización: comparación entre las cuatro promociones estudiadas (a) y los colegiados del COMB (b).

\section{a}

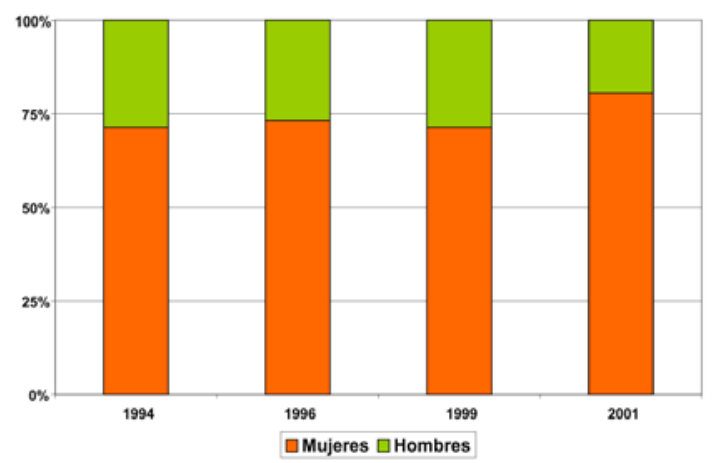

b

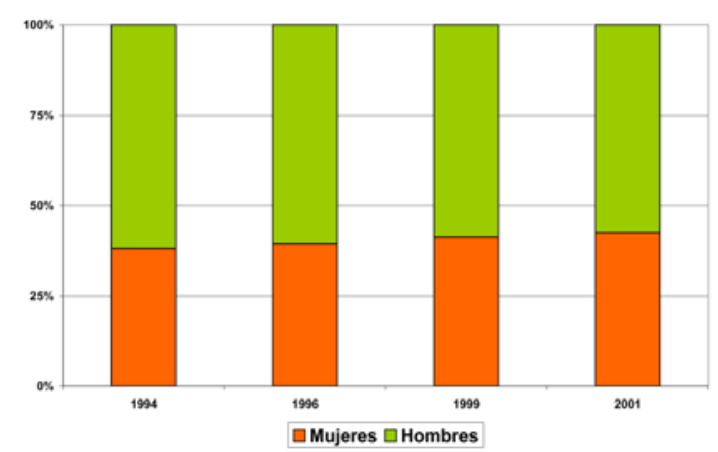

alumnos que acceden han tenido un buen rendimiento en la educación secundaria, que se refleja en una elevada nota de acceso, pues son estudios altamente demandados. Esta tendencia se confirma en 2011 (1.830 alumnos preinscritos en medicina de la UB como primera preferencia) [15]. El perfil académico incluye buenos hábitos de estudio, elemento imprescindible para su futura formación continuada como profesionales médicos.

La implementación del EEES ha conllevado el aumento de la oferta de plazas de medicina en toda Cataluña, debido principalmente a la puesta en marcha de tres nuevas facultades. En el curso 20102011, el número total de plazas fue de 1.044 entre públicas y privadas. En la preinscripción de junio de 2010 se unificaron los accesos de alumnos procedentes de bachillerato y de ciclos formativos de grado superior, poniéndolos así en competencia directa. El numerus clausus fijado para la UB se ha incrementado y para el curso 2011-2012 se ofertaron oficialmente 259 plazas. Sin embargo, su peso
Figura 4. Tasa de extranjería: comparación entre las cuatro promociones estudiadas (a) y los colegiados del COMB (b).

a

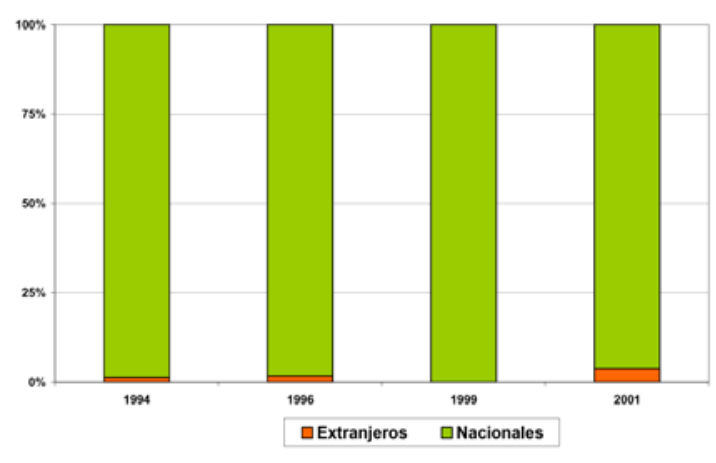

b

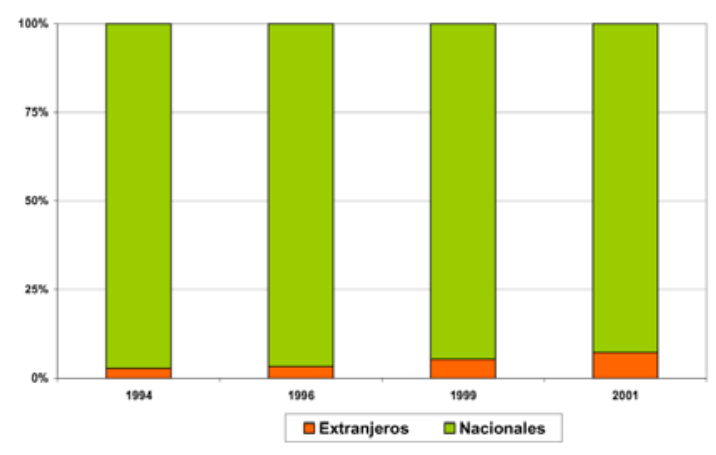

específico ha disminuido respecto a décadas pasadas y actualmente se sitúa en un $25 \%$.

La Facultad de Medicina de la UB sitúa a titulados bien preparados académicamente en disposición de afrontar la formación especializada, con una baja tasa de abandono de los estudios en las promociones estudiadas (4-13\%). Las tasas de licenciados en dichas promociones de medicina son siempre las más altas al compararlas con la treintena de estudios de primer y segundo ciclo de la UB. Además, la de medicina es la carrera con menor exceso en la duración de los estudios, con excepción de alguna filología [12]. Datos analizados con posterioridad al estudio han resultado similares para la promoción que se inició en 2003. Esto se traduce en unos indicadores de eficiencia muy próximos al $100 \%$. Sin embargo, debe tenerse en cuenta que altos resultados académicos no suponen automáticamente buenas carreras profesionales, en que son necesarias habilidades y capacidades de muy diversa índole, además de conocimientos. Esta constatación ha llevado 
a que la evaluación de competencias sea un tema de actual interés en educación médica [16].

La especialización es una salida mayoritaria y unas pocas especialidades absorben a la mayoría de titulados. Existen ciertas evidencias publicadas de una relación entre especialidades y sexo. Medicina familiar y comunitaria, obstetricia y ginecología, pediatría y psiquiatría, especialidades todas ellas con una alta importancia de la empatía, se han feminizado globalmente. La cirugía general y del aparato digestivo, así como otras especialidades quirúrgicas, continúan siendo elegidas tres veces más por hombres que por mujeres [17]. Por encuestas realizadas entre los alumnos de primer curso de las dos nuevas promociones (2009-2010 y 2010-2011) se evidencia que la medicina de atención primaria no está entre sus expectativas de futuro [18]. Por tanto, parece necesario reforzar el interés de los alumnos en este ámbito, dada su importancia en el actual sistema sanitario.

La incorporación a la profesión de los titulados ha sido buena, como indican las altas tasas de colegiación en la provincia. El sistema de salud, por tanto, ha tenido la capacidad y el atractivo para retener a los profesionales aquí formados. El peso relativo en las nuevas colegiaciones de los formados en la UB ha descendido del $25 \%$ al $12 \%$ en casi diez años por la afluencia de médicos formados en el extranjero.

Es probable que la crisis económica sea un freno del ciclo expansivo de nuevas plazas de pregrado y de MIR que hemos visto en los últimos años. Finalmente, habrá que comprobar si los resultados de un estudio reciente [19], que muestra que el $51 \%$ de los estudiantes de los últimos cursos de algunas universidades españolas desean emigrar, tendrán reflejo en la realidad futura.

La feminización de los titulados en medicina por la UB es una evidencia desde hace tiempo [20]. Esta elevada presencia femenina lleva progresivamente a cambiar la faz de la profesión, de manera que probablemente sea cuestión de pocos años el hecho de que el número de mujeres supere al de hombres entre los profesionales colegiados. En el año 2010, la cifra de mujeres colegiadas alcanzó el 47,6\%, y el $58,3 \%$ de nuevos colegiados fueron mujeres [21].

La presencia de alumnos extranjeros es mínima entre los titulados en la UB. Los numerosos profesionales extranjeros que se han colegiado se han formado, casi en su totalidad, en países extranjeros. Ello ha provocado en estos años paradojas como una demanda de estudios de medicina que no puede ser atendida por la universidad y, en paralelo, la llegada de profesionales de otros países que han venido a cubrir demandas del mercado laboral médico.
La determinación de las necesidades de médicos de manera fiable está sujeta a numerosas dificultades. En primer lugar, el período entre que un estudiante inicia su formación y se sitúa en el mercado de trabajo con su especialización finalizada no es inferior a 10-12 años. En segundo lugar, la gran mayoría de las variables predictivas del número adecuado de profesionales están sujetas a cambios que hacen muy difícil determinar la mencionada cifra. La afluencia migratoria, por ejemplo, resultaba francamente difícil de prever a inicios de este milenio. Lo mismo podemos decir de otras variables que pueden afectar directamente al número necesario de profesionales: los ciclos económicos expansivos o recesivos, los avances tecnológicos y los cambios en la cartera de servicios.

Parece prudente la política seguida hasta el momento por las autoridades catalanas de aumentar ligeramente el número de plazas para el estudio de la medicina, puesto que moverse entre dos extremos puede tener muy negativos, y ya conocidos, efectos sobre el sistema: falta de profesionales o cohortes de graduados sin posibilidad de acceder a la formación especializada.

En la formación de grado hay que hacer una apuesta estratégica pensando en profesionales de futuro con un mayor equilibrio entre habilidades y aptitudes, aspectos claves en el ejercicio de la medicina [7]. La admisión en primer curso debe seguir siendo exigente, pero podría ser más flexible añadiendo criterios que den más oportunidades a estudiantes que deseen cursar medicina.

Como conclusión final del estudio afirmamos que, en el marco actual, la Facultad de Medicina de la UB cumple con la función de formar profesionales que estén en condiciones de integrarse como tales en la sociedad catalana.

En la línea de formación integral y flexibilidad, opciones como la plena implantación y desarrollo del EEES en la formación de grado, por un lado, y de los aspectos previstos en la Ley de Ordenación de las Profesiones Sanitarias (troncalidad, áreas específicas de capacitación, programas pasarela entre especialidades) [22] en la formación de especialistas, por otro, deben ayudar a completar unas cohortes de médicos plenamente preparados para afrontar las situaciones cambiantes de la profesión médica.

\footnotetext{
Bibliografía

1. Proceso de Bolonia. Wikipedia. URL: http://es.wikipedia. org/wiki/Proceso_de_Bolonia. [01.03.2011].

2. Oficina de l'Espai Europeu del Coneixement. Educació, competitivitat i ocupació a Europa. Barcelona: Consell Interuniversitari de Catalunya; 2005.
} 
3. Prat J, Carreras J, Branda L, Miralles R, Fenoll, MR, Rodríguez S, et al. Competencias profesionales básicas comunes en los licenciados en Medicina en las universidades de Catalunya. Barcelona: Agència per a la Qualitat del Sistema Universitari de Catalunya (AQU); 2004

4. Roy-Schwarz M. Misión de las facultades de medicina en el siglo XxI. JANO 2008; 1701: 12-7.

5. Aunión JA. Empleabilidad es la asignatura pendiente. El País, 31 de mayo de 2012

6. Orden ECI/332/2008, de 13 de febrero, por la que se establecen los requisitos para la verificación de los títulos universitarios oficiales que habiliten para el ejercicio de la profesión de médico. BOE, 15 de febrero de 2008

7. Gual A, Oriol-Bosch A, Pardell H. El médico del futuro. Med Clin (Barc) 2010; 134: 363-8.

8. Real Decreto $1125 / 2003$, de 5 de septiembre, por el que se establece el sistema europeo de créditos y el sistema de calificaciones en las titulaciones universitarias de carácter oficial y de validez en todo el territorio nacional. $\mathrm{BOE}$ 18 de septiembre de 2003.

9. Campos B, Abellana R. Uso del campus virtual para evaluación presencial acreditativa. Educ Med 2011; 14 (Supl 1): S19.

10. Marco de verificación, seguimiento, modificación y acreditación de títulos oficiales. Barcelona: Agència per a la Qualitat del Sistema Universitari de Catalunya (AQU), Consell de Direcció, Generalitat de Catalunya; 2010.

11. Informe preliminar sobre la governança universitària. Projecte governança UB. Barcelona: Universitat de Barcelona, Comissió de Governança; 2011.

12. Portal estadístiques, planificació acadèmico-docent de la UB. URL: http://www.ub.edu/dades_academiques/index.htm.

13. Ministerio de Sanidad, Política Social e Igualdad. Formación sanitaria especializada. URL: http://sis.msps.es/fse/Default. aspx?MenuId=IE-00.

14. The R project for statistical computing. 2011. URL: http:// www.r-project.org/.

15. Playà J. Crece el interés por la universidad. La Vanguardia, 23 de julio de 2011.

16. Prades A, Rodríguez S, Carreras J, coords. Guía para la evaluación de competencias en Medicina. Barcelona: Agència per a la Qualitat del Sistema Universitari de Catalunya (AQU), Generalitat de Catalunya; 2009.

17. Petrides KV, McManus, IC. Mapping medical careers: questionnaire assessment of career preferences in medical school applicants and final-year students. BMC Med Educ 2004; 4: 18.

18. Campos B, Ornelas A, Cussó R. Comparación del alumnado de primero de los grados de Medicina e Ingeniería Biomédica. Educ Med 2011; 14 (Supl 1): S18-9.

19. Bernardini-Zambrini D, Barengo N, Bardach A, Hanna M, Macías-Núñez J. ¿Migrar o no migrar? ¿Qué pasará con nuestra próxima generación de médicos? Estudio sobre causas y motivos en estudiantes avanzados de medicina en 11 universidades de España. Aten Primaria 2011; 43: 222-6.

20. Gómez A, Durán J, De Anta JM, Götzens V. La feminización de nuestras aulas. El caso de la facultad de Medicina-Bellvitge de la Universidad de Barcelona. Educ Med 2011; 14 (Supl 1): S26 [póster 24].

21. Col-legi Oficial de Metges de Barcelona (COMB). Informe anual. 2010. URL: http://www.comb.cat/cat/actualitat/ publicacions/anuari/anuari2010/pdf/demografia1.pdf.

22. Ley 44/2003, de 21 de noviembre, de ordenación de las profesiones sanitarias. BOE, 22 de noviembre de 2003. p. 41442-58. 